\title{
Nervous System Sponsor-Defined Identifier
}

National Cancer Institute

\section{Source}

National Cancer Institute. Nervous System Sponsor-Defined Identifier. NCI Thesaurus.

Code C162199.

One or more sponsor defined characters used to identify, name, or characterize the nervous system finding. 